\title{
Cumulative reporting of chemical pathology
}

\author{
F. V. FLYNN AND JILL VERNON \\ From the Department of Clinical Pathology, University College Hospital, London
}

SYNOPSIS A simple system for producing cumulative chemical pathology reports is described Results of tests are transcribed onto the patient's laboratory record card and each time a new result? is entered a copy is made by xerography and returned to the clinician as the laboratory report. $\quad \vec{\infty}$

A detailed account is given of the operation of the system and the design of the record card. Their advantages of the system are enumerated and various problems that have been encountered are $\overbrace{\infty}$ discussed.

In reporting the findings of laboratory investigations it is usual for the pathologist to issue a form which carries only one result, or at most a small group of results. As a consequence numerous reports may accumulate in one patient's notes and however well these are filed it becomes difficult for the clinician to get an overall picture of the pathological findings. If reports are filed separately in the laboratory as well a great deal of storage space is required and it is not easy for the pathologist to compare the latest findings with earlier ones. The system to be described of reporting laboratory results on a cumulative basis was designed to overcome these difficulties, which will inevitably increase as more and more investigations are carried out. It has been in use for chemical pathology at University College Hospital, London, for a period of six months. The results of all the biochemical investigations on a given individual are entered on a printed card which is kept in the laboratory. Each time a new result or batch of results is entered, a copy of the card is made by xerography and despatched to the clinician as the laboratory report. Only the latest copy is retained in the patient's notes as it supersedes all previous reports.

\section{DETAILS OF SYSTEM}

GENERAL PROCEDURE Requests for chemical pathology are made on the usual type of form but the patient's identification data and location are all entered in a box at the top right-hand corner. In this box there is space for the patient's surname, first names in full, case number, and date of birth, as well as the ward or department.

The request form circulates in the laboratory and Received for publication 23 April 1965. the technician writes the result of a test on the lowero half and returns it to the secretary. The secretary has $\mathbb{\Phi}$ in the meantime found the patient's record card in the laboratory file, or made out a new one by entering the identification data. She checks or $\vec{\oplus}$ amends the pencilled entry of ward and clinician, types in the latest result, and gives the completed card and request form to the pathologist.

The pathologist checks the identification data, the destination of the report and the accuracy of the typist's copying, and reviews the latest entry in the $\frac{}{\mathbb{Q}}$ light of the clinical information and the results of all the previous investigations. If necessary heo를 queries a result, asks for a correction to be made, or 3 writes in a comment, and when satisfied he hands the card back to the secretary for xerographic copying. The pathologist's signature and the date of copyingo are stamped on the xerographic report before it is despatched, the latter entry readily identifying the latest report for the clinician.

THE CUMULATIVE RECORD CARD The cumulativeo record is made on 'two-sheet' card, which is robust? enough to stand repeated handling and sufficientlyo flexible to feed into a typewriter. The overall size of the reports had to be compatible with the size of theos patient's notes (10 in. $\times 8$ in.), but the exactro dimensions of the card were determined by the sizen of the scanning plate of the copier and the necessity of copying two cards simultaneously to keep the cost of the process within reasonable bounds. The maximum size of the card worked out at $10 \mathrm{in.} \times \mathbb{\Phi}$ $7 \frac{5}{8}$ in., the printed area being $9 \frac{1}{4}$ in. $\times 7$ in. as the outer $\frac{1}{4}$ in. or so of the scanning plate is not functional ${ }_{0}^{-}$ A $\frac{5}{8}$ in. margin was left clear at the top of the card sod that the copies can ultimately be filed with the notes in book form, and the bottom left-hand corner 


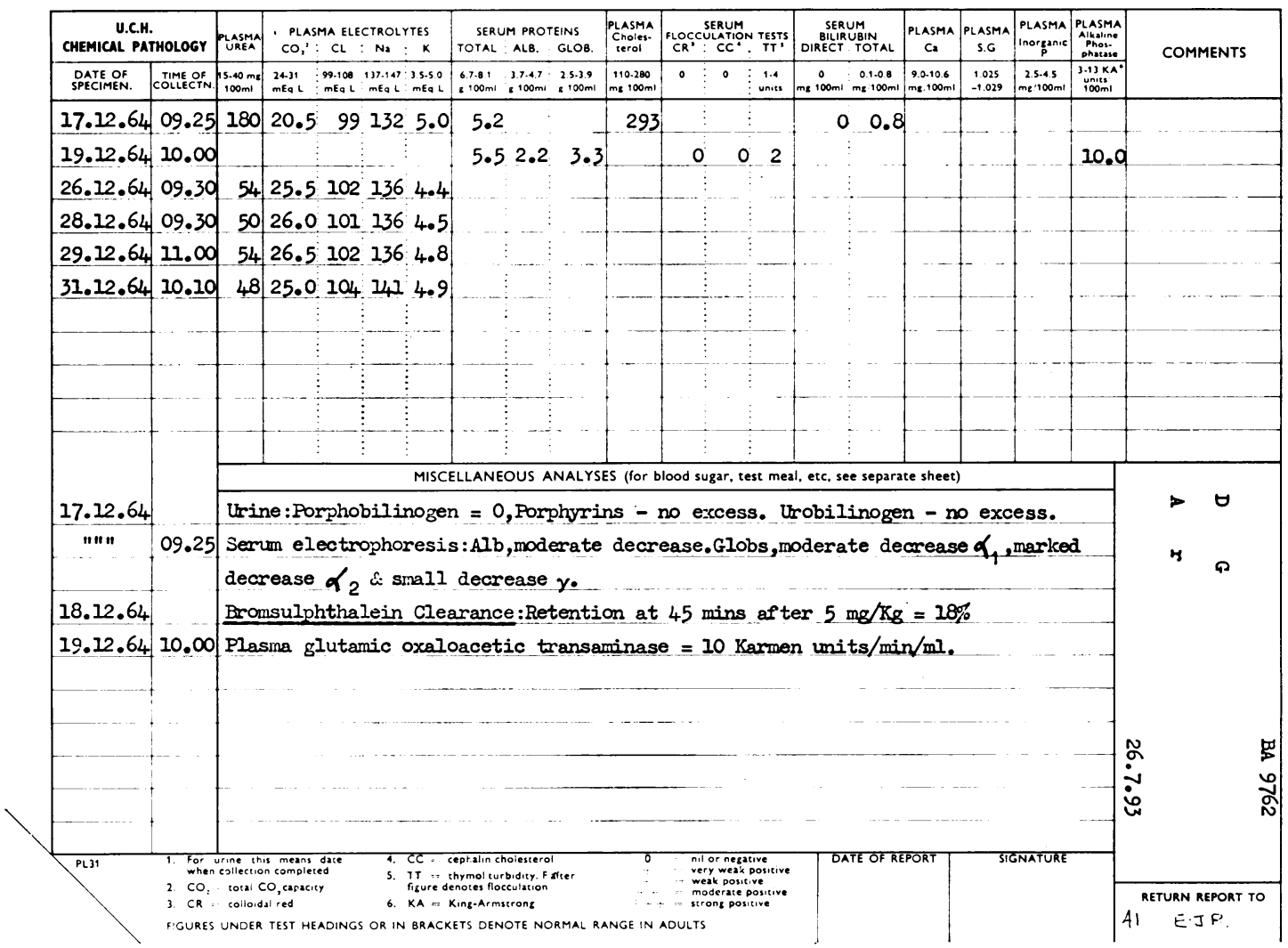

FIG. 1. Front of the printed area of a cumulative record card showing layout and use. The patient's name has been partly obliterated.

was marked off also to conform with future requirements of the Records Department. Quarter inch margins were left at the sides, as these lie outside the image area of the scanning plate, but printing was continued right down to the lower edge of the card as this is always placed to the centre of the plate when two cards are being copied simultaneously. The front and back of the card are tumbleprinted so that it can be turned easily when both sides are to be copied.

The front of the card is shown in Figure 1. It is designed to hold as many results as possible in the space available, the more frequently performed tests being allocated specific columns which are grouped into convenient categories, while the less common tests are entered under a 'Miscellaneous' heading. Each column has the normal range and the units printed at the top, which saves repetition of these items. The width of the columns was planned for use with a typewriter which prints 12 characters to the inch, and each column will just accommodate the largest number of digits possible for that particular estimation. Once lined up on one of the vertical lines a whole row of figures can be typed accurately into the columns. The space between lines was selected so that the maximum number of results could be recorded without sacrificing clarity or being too demanding on lining up by the typist. A test result under 'Miscellaneous' can usually be accommodated on one line.

The back of the card, shown in Fig. 2, is intended to be versatile and accommodate results of tests which may take up a lot more space than could be allocated on the front of the card. Printed labels have been designed to stick onto one or more of the five equal-sized boxes according to requirement, and at present there are labels for blood sugars, which when repeated are liable to be very numerous, gastric analysis, and blood sugar curves, the last occupying two boxes; others may be added in the future.

The identification box on the front of the card is placed so that the card can be filed with the long axis vertical. There is space for the same information 


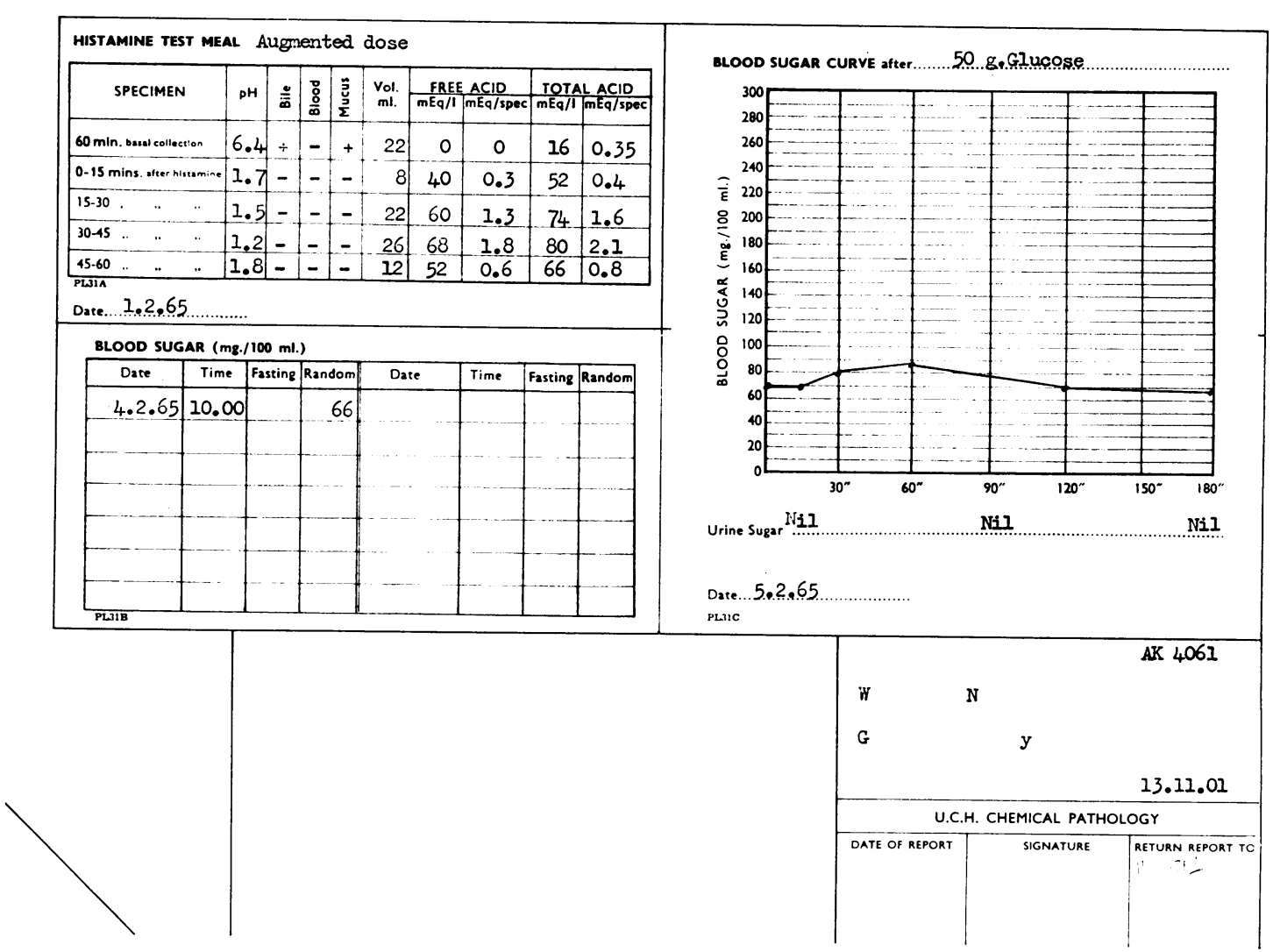

FIG. 2. Back of the printed area of a cumulative record card with printed labels attached, showing layout and use. The patient's name has been partly obliterated.

as is given in the corresponding box of the request form, and the dimensions ( $3 \frac{1}{4}$ in. $\times 1 \frac{1}{4}$ in.) are such that an Addressograph label may be used to supply this information in the future. When entries are made on the back of the card a similar box has to be completed because the two sides are copied onto separate sheets of paper.

In order to save space some abbreviations and conventions are used in reporting results but these are all explained in the footnotes on the front of the card. For instance with 24-hour urine collections only the date of completion of the collection is noted, and for the results of qualitative tests there is a set of five signs ranging from negative to strong positive. To save writing a.m. and p.m., the 24-hour clock is used when entries are made in the 'time of collection' column. The 'comments' column is for the pathologist to make any special notes about the specimen or the result. The destination box, headed 'return report to' is for entry of the ward or clinic and the consultant's initials; it is filled in in pencil so that alterations can be made if need be. The boxes for 'date of report' and 'signature' are only used on the xerographic copies.

XEROGRAPHIC COPYING The copying equipment used in the laboratory is the Rank-Xerox 914 Office Copier, which is a free-standing unit requiring $7 \mathrm{ft} . \times 7 \mathrm{ft}$. of clear floor space. It is simple to operate and by a dry process makes excellent copies ready for immediate despatch; it produces almost equally $N$ good images of ink, ballpoint pen, pencil, typescript, and print. For the present work a template has been designed to fit over the glass scanning plate so that two record cards can be placed accurately upon it to be copied simultaneously. This template is shown in Figure 3.

Before the copying process can begin there is a warming-up period of about five minutes. During 0 this time the operator can clean the scanning plate, load up the machine with the paper and stir up the black 'toner' powder used in the copying process, 


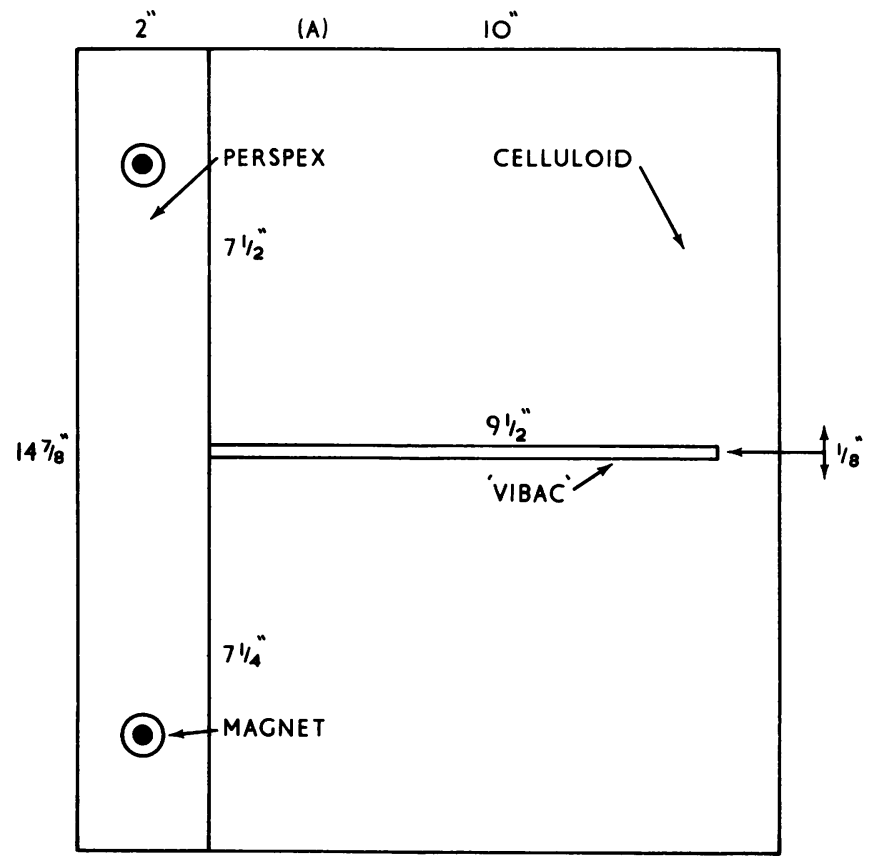

FIG. 3. Diagram of template used for positioning cumulative cards on scanning plate of copier; (a) view from above, (b) end-on view.

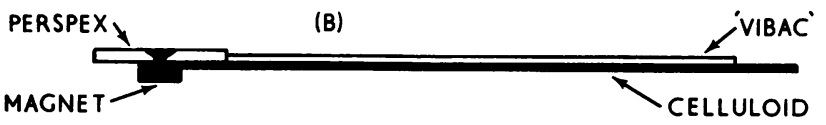

all of which are daily necessities. Two cards are then laid side by side on the template and the 'print' button is pressed. Scanning begins immediately and in 6.5 seconds the signal to re-load becomes illuminated. The sheet of paper bearing images of the first two cards appears in the output tray in about 30 seconds and with rapid reloading, succeeding reports follow at a rate of up to three pairs a minute. Once the procedure has started the operator is kept fully occupied supplying the machine with cards, collecting the copies as they appear, tearing them down the centre along a pre-slotted line, stamping them with the date and stapling together any paired copies.

The cards are placed face downwards on the scanning plate, with the bottom edge of the side to be copied lying to the centre of the plate. When so orientated the corner mark on the reverse of the card lies away from the centre of the plate and this has proved a useful guide to the operator when positioning cards at speed. Whilst one side of the card is being copied it is possible to see if the other side needs copying too, and the tumble-printing makes it a simple manoeuvre to reverse the card if necessary.

The paper used for the copies is loaded into a drawer in the base of the copier. It must be of good quality and kept absolutely dry and for the present work sheets $15 \frac{1}{2}$ in. $\times 10$ in. are used; this paper is slotted so that it can be easily separated after the copying process into two pieces measuring 10 in. $\times$ $7 \frac{3}{4}$ in. Before loading, the stacks of paper are 'fanned' by curving them over first in one direction and then in the other to make sure that the sheets are not adherent to each other.

The Xerox copier is rented by the month, the rental depending on the number of copies made during that period; the minimum charge is $£ 30$, which includes the cost of making, 2,000 exposures i.e., 4,000 single-sheet reports. Servicing comes under the rental charge but the cost of paper, 'toner' powder, cleaning fluid, rayon wool, and electricity are all additional items. Taking all these into account the cost of producing a single-sheet pathology report works out at $2 \cdot 25 \mathrm{~d}$. Credit is given on the monthly account for faulty copies arising as a result of machine errors.

Running a Xerox copier entails a few maintainance chores, including the periodic cleaning of the selenium drum which is a delicate operation. Major 
breakdowns have not occurred but minor troubles have been encountered intermittently and not infrequently. Failure to pick up copy-paper is an annoying problem when working at speed, as it is easy not to notice that a copy of a particular pair of cards has not been made. By comparison, the taking of duplicate copies when only one has been dialled is easily spotted and is of little consequence. The jamming of paper in the machine calls for its urgent removal, which may be a dirty, difficult and timeconsuming job.

\section{DISCUSSION}

A cumulative laboratory report has important advantages over a series of individual reports. It saves the clinician time by giving him a concise résumé of all the pathological findings in date sequence, and if he should require another copy of the report it is easy to supply one. The pathologist too can rapidly gain a picture of the laboratory findings and compare the latest result with previous ones, which is a valuable form of quality control. Less filing space is needed in the laboratory and the information on the record cards is readily available for analysis. Finally, the Records Department benefits from the decreased bulk of pathology reports and the availability of further copies for summaries. At University College Hospital, cumulative reporting has so far been used only for chemical pathology, but the advantages of the system would apply equally to the other branches of clinical pathology.

Operation of the cumulative reporting system requires that the laboratory is absolutely certain of a patient's identity. If results are entered on the wrong card it may have serious consequences, and if a new card is started for a patient who already has one, the rule to retain only the latest copy in the notes will lead to previous results being discarded. Whereas in other systems it is usual for staff in ward or outpatient clinic to place the latest report adjacent to the previous ones, with the new system the onus of doing this falls on the laboratory where there is greater risk of confusing individuals of the same name. Theoretically, the use of the hospital case number should prevent mistakes occurring, but in practice this is not always available or it may be incorrectly reproduced and therefore it is necessary to ask for additional information on the request form, for example forenames in full and date of birth. It is hoped that in the future the problem of identification will be solved when all request forms will be marked with an Addressograph label, made out for each patient on registration.

No extra staff have been required to run the new system but additional responsibility has been thrown

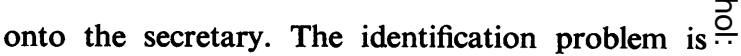
chiefly hers and she is solely responsible for the $\overrightarrow{\vec{F}}$ accurate filing of record cards so that new ones are not started erroneously. Her peak of activity occurs towards the end of the day when most of the typing and xerography have to be done, and $\vec{\nabla}$ initially this necessitated a small adjustment of her $\cong$ working hours. The typing, though exacting, is less $\tilde{\omega}$ tedious than formerly as in the majority of cases $\vec{\circ}$ only figures have to be entered. It is not essential to have results typed but it is clearer and neater; when $\vec{\omega}$ no secretary is available results for the current $\frac{\circ}{2}$ report are entered on the card in pencil and typed? in at a later date. The cards are not allowed to $\vec{\infty}$ circulate in the laboratory as they would inevitably or become soiled and problems would arise as a result of their dispersal.

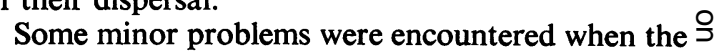
system was introduced. It took a little time to get $\vec{c}$ used to the layout of the report, and the nursing $\mathbb{D}$ staff had to overcome their reluctance to throw away $\frac{\vec{D}}{\mathbb{D}}$ earlier versions. Making clear corrections on the $\stackrel{3}{3}$ master card proved difficult until it was discovered that self-adhesive labels could be used to cover over any errors. A serious problem remained in that the $\mathscr{\varphi}$ whole system would be disrupted if there was a major breakdown of the Xerox copier. The makers provide a good repair service but to cover all eventualities we have made a reciprocal arrangement with nearby users of a similar machine whereby either can use the other's copier if necessary. Minor $\mathbb{D}$ hitches, such as arise from copy-paper sticking, have $\overrightarrow{\overrightarrow{0}}$ not been infrequent, but these, though irritating, 3 can be put right by any competent operator who has $\vec{F}$ been instructed by the Rank-Xerox demonstrator.

It is not new to use xerographic copying to produce pathology reports. In this country Vickers? and Fitzpatrick (1963) and the Reading and District $\frac{}{3}$ Group Pathology Department (Abstracts of Efficiency Studies in the Hospital Service No. 72, 1964)윽 have used it as an alternative to copy typing or photocopying for duplicating individual reports, ? and it was found to save time and tedious work and $>$ slightly to reduce the cost of each report. Xerography used in this way, however, seems to have little $N_{\text {S }}$ advantage over the use of printed N.C.R. forms, by which all entries on the top sheet of a combined request and report form are automatically dupli- $\omega$ cated onto a similar undersheet by a dry chemicalo process. Mason (1962) at the Medical Center, Rochester, N.Y., has used xerography to produce a cumulative report very similar to the one we ${ }^{?}$ describe, but his record card had a series of blank ${ }^{T}$ columns. Adhesive labels printed with the different tests and the relevant units were affixed to the headso of the columns as required, and results were entered $\stackrel{\mathbb{Q}}{\Omega}$ 
by the technician undertaking the test. A printed card such as we employ has the advantage that the format becomes familiar and time is not wasted searching for a result. A very similar system to the one we describe has been devised independently at the Edinburgh Royal Infirmary (Whitby and Owen, 1965).

The cumulative system has now been in use for six months and the design of the record card has proved very satisfactory for present requirements. When the cards of 1,000 patients were analysed after five months' use, it was found that 939 patients had entries on one side of the card only, and that only 15 patients had entries on more than one card, nine of these having filled the top section and six the miscellaneous section. At five months the total number of investigations on these cards was 6,992 and of these 946 were under the miscellaneous heading. The number of investigations per patient varied between one and 264 .

The cost of producing each single-sheet report is appreciably greater (approximately by $1.25 \mathrm{~d}$ ) with the xerographic process than with the N.C.R. forms previously used, but the advantages of the cumulative system seem to justify the added expense. At present when one card has been filled with results and a second one started, copies are made of all the card surfaces bearing results and all these copies are stapled together as the latest report. This is clearly a wasteful procedure, but it was devised so that the rule about keeping only the latest report in the notes would be easy to apply. At present few patients require two- or three-sheet reports but in the future it is intended to mark filled cards with a 'file permanently' label, so that only the current card will need to be copied.

We wish to acknowledge valuable help received from Mr. P. J. Somerville of Rank-Xerox Ltd., in devising the template and from Miss G. Richards for analysing the record cards. This work was supported from research funds granted to University College Hospital by the Ministry of Health.

\section{ADDENDUM}

Since submitting this paper for publication a timesaving modification has been made to the method of adding the date and pathologist's signature to the final report. Instead of stamping these on the individual xerographic copies, labels carrying these items are now fixed with Sellotape face-down on the template in positions corresponding to the appropriate boxes on the record card. Since these boxes are in the same relative position on the front and back of the cards the labels need only be placed in position once each day.

\section{REFERENCES}

Abstracts of Efficiency Studies in the Hospital Service, no. 72. (1964.) H.M.S.O., London.

Mason, W. B. (1962). Ann. N.Y. Acad. Sci., 102, 171.

Vickers, H. E., and Fitzpatrick, J. W. (1963). Hospital (Lond.), 59, 627.

Whitby, L. G., and Owen, J. A. (1965). J. clin. Path., 18, 668. 